Voix et Images

\title{
La Préciosité dans le théâtre politique de Jacques Ferron
}

\section{Jonathan M. Weiss}

Volume 3, numéro 1, septembre 1977

Nicole Brossard

URI : https://id.erudit.org/iderudit/200093ar

DOI : https://doi.org/10.7202/200093ar

Aller au sommaire du numéro

Éditeur(s)

Les Presses de l'Université du Québec

ISSN

0318-9201 (imprimé)

1705-933X (numérique)

Découvrir la revue

Citer cet article

Weiss, J. M. (1977). La Préciosité dans le théâtre politique de Jacques Ferron.

Voix et Images, 3(1), 127-146. https://doi.org/10.7202/200093ar d'utilisation que vous pouvez consulter en ligne.

https://apropos.erudit.org/fr/usagers/politique-dutilisation/ 


\section{La Préciosité dans le théâtre politique de Jacques Ferron}

On a eu du mal à caractériser les deux pièces politiques de Jacques Ferron (les Grands Soleils et la Tête du roi). André Vanasse, par exemple, trouve que les pièces non politiques de Ferron se placent tout naturellement sous le signe du «cycle d'imitation de la comédie classique", mais ce critique se heurte à une impasse lorsqu'il veut donner un titre à la "deuxième période" (la période politique) dans la dramaturgie de l'auteur ${ }^{1}$, et il en conclut que "Ferron est incapable de traiter théâtralement un sujet politique", conclusion qui sonne faux après le succès des Grands Soleils en 1968 et sa reprise en 1977². Pour Guy Beaulne, ce théâtre politique s'apparente à celui de Brecht par son «insolence", son goût pour "le dépaysement" et son “désir d'éveiller la conscience populaire aux injustices sociales et politiques ${ }^{3}$ ". Dans la même ligne, Jean-Marcel Paquette voit le désir chez Ferron d'établir "une 'distanciation' de type brechtien" et souligne la «fonction critique permettant la réflexion sur la théâtralité » de tout le théâtre de l'auteur ${ }^{4}$. Enfin, Michelle Lavoie met en relief le caractère essentiellement littéraire de la dramaturgie ferronnienne et suggère qu' "en dépit du modernisme du vocabulaire et du contexte, c'est à un certain $x^{\prime \prime I I}{ }^{e}$ siècle français que nous fait penser Jacques Ferron ${ }^{5}$ ».

Sans entrer ici dans une discussion du mérite de ces jugements critiques, il est remarquable de noter qu'il y a un mot qui revient souvent quand on parle de Ferron mais qu'on n'a jamais appliqué de façon systématique à son théâtre: celui de préciositéb. Ce mot, dont les origines remontent au $\mathrm{XVII}^{\ominus}$ siècle mais qui a été redéfini et remis à jour en France par Claude-Edmonde Magny, a maintenant un sens beaucoup plus philosophique qu'esthétique: il indique un mode d'écrire où règnent des archétypes, des essences, «dont chacune est douée d'une structure individuelle et qui constituent les principaux types d'organisation du réel ${ }^{7}$ ". ClaudeEdmonde Magny a utilisé le mot précieux pour parler surtout de Giraudoux, se basant sur un article que Sartre a écrit en 1940 et où il accusait Giraudoux de ne pas pouvoir s'adapter au réel ${ }^{8}$. Mais l'on peut, à mon avis - même sans proposer la moindre influence de Giraudoux sur Ferron - voir dans le théâtre ferronnien la même organisation du réel 
en un monde d'archétypes, de perfections, qu'on voit communément chez l'auteur de La Guerre de Troie n'aura pas lieu.

J'entends montrer, dans les pages qui suivent, que la notion de la préciosité, telle que l'a définie Madame Magny, est la clé à la compréhension du théâtre politique de Ferron. Pourquoi son théâtre politique? Parce que, sans nier l'application éventuelle de ces concepts à tout son théâtre et même à toute son œuvre, c'est le contexte proprement politique qui fournit le meilleur terrain pour le heurt des forces opposantes qu'entraîne le théâtre précieux. Et surtout parce que la préciosité a une importance profonde quant à la façon de voir le monde politique, ce monde composé de groupes et de factions, de majorités et de minorités qui imposent à l'homme une identité qu'il doit reconnaître avant de pouvoir revendiquer son droit d'exister et de s'épanouir.

\section{La Préciosité}

Résumer en quelques paragraphes l'idée de la préciosité mise en avant par Claude-Edmonde Magny n'est pas chose simple. Essayons de dégager les points essentiels de son argumentation, ceux qui sont le plus susceptibles de s'appliquer à Ferron.

La conception qui sọus-tend l'idée de la préciosité est celle de l'immobilité, du caractère inchangeable de toute situation; dans le monde de Giraudoux, affirme Madame Magny, «il n'y a ni casualité efficiente ni devenir temporel, " et “le seul changement imaginable est la réalisation, chaque jour un peu plus parfaitement par chaque chose, de son essence» (p. 21). Sartre avait dit à peu près la même chose en disant que, dans l'œuvre de Giraudoux, "les personnages par leurs actes, les choses par leurs changements ne font que réaliser plus étroitement leur forme substantielle» (p. 80). La substantialité (dans son sens philosophique qui implique la permanence et qui est le contraire de l'accidentel) confère à la préciosité son caractère le plus remarquable: tous les aspects composant le monde se réunissent dans l'affirmation de leur cohérence. Dans un tel univers, rien ne surprend. L'apparence de l'objet, du personnage, nous révèle son essence, et rien ne détruit cette unité profonde de l'être et du paraître. Comme l'affirme Sartre, «il y a une convenance intime de l'homme avec le monde" (p. 86). Cette convenance est encore plus marquée au théâtre que dans la prose, car le théâtre fait appel aux yeux et aux oreilles d'un public qui doit saisir le rapport entre le signe théatral (le mot prononcé, accompagné d'effets visuels et sonores) et une signification qui n'a, au fond, que très peu de rapport avec le monde imparfait où nous vivons. Le propre du monde précieux est d'être parfait; si Ondine, les dieux grecs, ou le Spectre incarnent, chez Giraudoux, deș esprits divins qui voient ce que les hommes ne peuvent pas voir, Hans, Hector, et le Contrôleur n'en sont pas moins parfaits dans leur caractère d'hommes qui est de faire des erreurs et de ne pas pouvoir prédire l'avenir. Ainsi la préciosité implique un univers fictif créé de toutes pièces pour 
correspondre à un certain système de pensée. Quand on a compris ce système, on a tout compris.

Parmi les techniques stylistiques employées dans la construction de l'univers précieux, la plus importante est sans doute l'antithèse. Ce procédé, que Madame Magny qualifie de «transposition exacte des 'alliances de mots' chères à Voiture et à Benserade» (p. 33) a l'avantage de «rendre plus manifeste l'essence d'un objet par l'opposition avec ce qui n'est pas lui » (p. 34). Chez Giraudoux, bien sûr, ces antithèses abondent. On n'a qu'à penser à Siegfried et à Jacques (Siegfried), chacun représentant l'essence de leur pays respectif, ou à Alcmène et à Jupiter (Amphytrion 38), si parfaitement dieu et femme, pour voir comment une antithèse peut être à la base d'une pièce théâtrale. Les personnages, se heurtant à leur contraire, deviennent plus facilement des archétypes; leur essence se détache avec d'autant plus de relief qu'on ne les voit jamais seuls. Ainsi, dans le monde précieux, tout existe par rapport à son contraire, ou, comme l'explique Madame Magny:

L'antithèse est l'analyse chimique grâce à laquelle le précieux réussit à séparer effectivement les uns des autres les corps simples qui forment le monde, essences indestructibles que la réalité nous montre d'ordinaire mêlées, réciproquement souillées. (p. 36)

Cette confrontation d'archétypes nous amène tout naturellement à considérer le deuxième procédé stylistique qui définit la préciosité: le caractère foncièrement insoluble des conflits. Si l'on a accusé Giraudoux de ne jamais conclure ses pièces, de toujours «s'esquiver par une pirouette aux moments cruciaux" (C.-E. Magny, p. 95), c'est que, nous dit Madame Magny, la préciosité exige «une impossibilité objective de mettre à une cuvre le point final." (p. 95). Saura-t-on jamais si c'est par haine patriotique ou par amour que Judith tue Holopherne? Tout ce qu'on peut en savoir est qu'en tuant Holopherne Judith a été fidèle à son essence qui est de s'opposer au général assyrien et de l'aimer en même temps.

Dans un monde d'archétypes, d'antithèses, de conclusions impossibles, il est inévitable que l'événement, l'action de la pièce, sera subordonné aux personnages. D'où la troisième et dernière caractéristique de la préciosité qui est d'évoquer une atmosphère de bal masqué. Les personnages se promènent dans cet univers derrière le masque de leur apparence, mais on a beau leur arracher ce masque, aucun visage n'est caché derrière. Sur le plan philosophique, donc, le bal masqué est la transposition esthétique de la notion de la substantialité ou, comme le souligne Madame Magny, «l'expression d'une vérité métaphysique profonde: l'impossibilité où nous sommes de connaître la vérité définitive sur quoi que ce soit » (p. 103).

On voit aisément que, malgré sa cohérence, l'univers précieux n'est pas simple. Complexités et ambiguités y abondent, et les masques résistent à une analyse facile. Des traits d'apparence contradictoires peuvent coexister au sein d'un même personnage ou d'un même objet. Ainsi, 
par exemple, Judith est à la fois amante et tueuse; Ulysse, bien que connu pour sa cruauté, est assez indulgent envers Hector. Mais ces ambiguïtés existent dans le cadre asseż restreint d'une essence bien définie: Judith est, sans la moindre équivoque, une Juive et Ulysse est aussi certainement un Grec. Mais que signifient ces essences? Voilà le problème que l'écrivain précieux essaie de résoudre, non pas en analysant ses personnages à la façon d'un psychanalyste mais en multipliant autour d'eux les images, les symboles, les formes et les couleurs qui semblent le mieux mettre en relief les aspects les plus signifiants de leur essence. La préciosité est donc le contraire de la transparence, et les mots employés par l'écrivain précieux sont d'autant plus significatifs qu'on ne passe pas facilement à travers eux. Laissons à Madame Magny le dernier mot: «L'emploi de la rhétorique précieuse se justifie finalement par l'ambiguïté fondamentale de l'univers qu'elle seule peut expliquer adéquatement. " (p. 117)

Dans les analyses qui vont suivre, je vais essayer de dégager pour chaque pièce, les caractéristiques qui me semblent le plus conformes à cette définition contemporaine de la préciosité. Aucun doute que la préciosité ferronnienne diffère considérablement de la préciosité giralducienne.

\section{Les Grands Soleils}

Cette pièce, dont je vais considérer la deuxième version, celle écrite pour la scène en $1968^{\circ}$, se révèle précieuse surtout par l'emploi que Ferron y fait d'images substantielles, ambiguës, opaques - images qui enveloppent les personnages et même le dialogue, et qui renvoient à une antithèse centrale qui sous-tend toute la pièce. Au cœur de cette imagerie on trouve le symbole principal de la pièce, l'hélianthe, le tournesol, le «grand soleil » du titre.

Ce «grand soleil» paraît comme le symbole de toute la population canadienne-française de l'univers de la pièce; comme le souligne Laurent Mailhot, “l'image du soleil-astre, du soleil-temps, et de la fleur-soleil, du soleil-espace, prend ici la valeur et la dimension d'un symbole collectif ${ }^{10}$ ". Parce que ces fleurs sont tournées vers le soleil, elles reflètent le visage d'Élizabeth quand elle raconte son rêve à Chénier, le matin; mais parce qu'elles symbolisent la chaleur, ces fleurs sont les flammes qui enveloppent l'église où, dans le rêve d'Élizabeth et plus tard dans la réalité, Chénier trouvera la mort. Simples plantes, les hélianthes sont les "grands yeux d'automne" (p. 40) qui «inclineront des fleurs aveugles» (p. 41) avant la bataille de Saint-Eustache; soleils couchants, à la veille de l'affrontement, ces «grands Soleils, gorgés de sang, éclateront au crépuscule" (p. 56). Les soleils sont à la fois les feux de bivouac des Patriotes et les étoiles dans le ciel; mais ils sont aussi l'ostensoir de l'église mise à feu par les Anglais et le feu du napalm lancé par des soldats canadiens au $x x^{\mathrm{e}}$ siècle. Enfin, ces tournesols signifient la vie rampante 
dans le jardin de Chénier «où les enfants boivent le lait des fleurs» (p. 67); mais, après la bataille perdue, ces fleurs ont «tourné à la graine et penchent vers la terre" (p. 76) et le soleil qu'elles reflètent n'est plus qu'une "grande corolle vide, une nacelle abandonnée, un astre mort et trompeur" (p. 76). De façon générale, on peut dire que les grands soleils ne sont jamais quelque chose sans être en même temps son contraire. Mais loin de s'anéantir, ces deux faces de la même image sont complémentaires. Si, d'après Mithridate, le soleil c'est Dieu «le Père, l'Amiral, l'Incendiaire, " si le soleil est le feu du four crématoire, du napalm, les victimes de cette destruction, les «pauvres Christs» qui «meurent toujours seuls et abandonnés" ont besoin de cet astre de la destruction; le Fils «n'est plus crucifié; il brûle vif pour que le soleil ne s'éteigne pas» (p. 100). Cette réciprocité de la vie et de la mort, de la mort nécessaire pour que renaisse la vie, Sauvageau en fait un résumé succinct: « $L a$ vie est chose étonnante, dit-il, il n'y a que la mort qui l'égale." (p. 89)

"Toute chose est en même temps son contraire, emportée qu'elle est... vers une signification autre que la sienne ${ }^{11 .}$ " Cette remarque que fait Claude-Edmonde Magny à propos de Giraudoux pourrait bien s'appliquer à Ferron. C'est que toutes les images des Grands Soleils sont si opaques qu'elles cessent de symboliser pour se coller à l'objet qu'elles représentent et le transformer ensuite en un objet extra-réel, théâtral par excellence. C'est ainsi que l'hélianthe ne peut, par son ambiguïté même, assumer aucune signification univoque; sa fonction est plutôt d'englober toute la pièce et de représenter, de plusieurs façons, chacune des étapes dans le développement de l'action et, par là, chacune des différentes consciences de la collectivité québécoise. D'autres images employées dans la pièce ont une fonction semblable. Considérons par exemple cette série de répliques dans la huitième scène du premier acte:

Mithridate: Et voilà qu'on commence à se décorer!

Sauvageau: Pas du tout! On se rubanne pour s'afficher et pour se reconnaître.

Mithridate: Et l'on est distingué, la belle affaire!

Sauvageau: Les Chouayens arborent le rouge, les Patriotes le blanc.

Mithridate: Le sang contre l'innocence. Le rouge attaque, le blanc se rend. Moi, j'aurais préféré le noir.

Sauvageau: Le rouge attaque, le blanc se défend mais, avant de se rendre, il virera au rouge. Ce sont des couleurs pour commencer.

Mithridate: J'aurais préféré le noir, c'est la seule couleur qui ne change pas.

Sauvageau: Regarde donc ton chapeau, coloriste!

Mithridate: Qu'est-ce qu'il a mon chapeau, Sauvageau?

Sauvageau: II est noir, mais il a tourné au vert on dirait. (p. 32) 
Si, au début de cet extrait, le rouge semble symboliser les Anglais et les Chouayens, et le blanc représenter l'innocence et la défaite des Patriotes, la suite des répliques rend impossible une si facile interprétation. Le blanc, apprend-on, "virera au rouge» avant de se rendre; Mithridate, dont la sympathie envers les Patriotes ne fait aucun doute, préférera le noir (couleur de l'oubli? de l'anarchie?) au rouge, mais, d'après le Sauvage, son noir est en train de tourner au vert (Mithridate "végète" - à moins qu'il ne s'agisse ici d'une plaisanterie mettant en cause la propreté de ses vêtements). Tout ce qu'on peut dire c'est que le rouge, avec ses significations multiples, est collé aux Anglais et à leurs alliés; que le blanc est attaché à la pureté des Patriotes, et que le noir, couleur non seulement de Mithridate mais aussi, à certains moments, d'Élizabeth (habillée de noir) et de Chénier ("le visage noir de poudre», p. 68), emprisonne dans un refus total ceux qui n'acceptent pas l'évolution de la situation historique.

On pourrait citer beaucoup d'autres exemples d'images qui, par leur ambiguïté, cessent d'être au service de l'action de la pièce pour la dominer. Même le décor semble avoir une existence qui dépasse les personnages qu'il contient. Jean-Marcel Paquette remarque avec justesse que le décor dans les pièces de Ferron se détache de la thématique interne "pour constituer un élément théâtral autonome, contaminant toute la pièce au point de lui donner sa véritable dimension ${ }^{12} \%$. Ferron nous assure, dans sa préface aux Grands Soleils, que la pièce «a été faite à partir d'un décor; celui-ci a donc de l'importance» (p. 11). A cheval sur deux époques - 1837 et 1968 - la maison de Chénier et son monument répondent à la gare Viger de Montréal; le jardin du docteur communique si librement avec le parc Viger qu' «il est difficile de savoir où l'un commence, où l'autre finit " (p. 11). L'importance de la gare Viger est d'autant plus curieuse qu'elle fut nommée, non pas pour le légendaire Vincent Bonaventure Viger (le «Beau Viger») de la pièce, celui qui a libéré les prisonniers de Colborne en se faisant arracher le bout du pouce gauche (p. 58) ${ }^{13}$, mais pour Jacques Viger, premier maire de Montréal, qui s'est battu à côté des Anglais pendant la Guerre de 1812. Ajoutons à cette liste d'homonymes Denis-Benjamin Viger qui, beaucoup plus modéré que son cousin Bonaventure, dut passer dix-huit mois en prison en 1837 à cause de l'appui, pourtant mitigé, qu'il donnait à Papineau. Lequel de ces trois Viger règne sur le décor? Inutile de le préciser. L'important, c'est que cette statue de Chénier, ce totem qui monte dans le ciel (p. 67) ainsi que la maison et le jardin du docteur sont entourés, dans toute la pureté de leur signification patriotique, par l'équivoque de la gare et du parc Viger.

Cette domination de la pièce par son décor et ses images est un des aspects les plus significatifs de la préciosité de l'œuvre. Comme dans Siegfried de Giraudoux, où l'Allemagne est représentée par le chauffage central et la France par le chauffage individuel, le Québec, dans les Grands Soleils, se résume dans le cri de ses femmes dont les maris ont quitté le foyer pour se battre contre les Anglais (cri à double sens puisque, 
pour le curé, il s'agit de rappeler le mari à la maison (p. 65) tandis que, pour Chénier, cet «appel d'une femme dans la nuit... qui fait vibrer le monde comme un grand violoncelle» ( $p .78$ ) est un appel à la guerre). Les Québécois sont des "braconniers, remplis d'étonnement" devant la guerre et Chénier, leur capitaine, «ne connaît que le sang des accouchées» (p. 74). Les Anglais, par contre, sont nés guerriers; ils portent l'uniforme rouge (couleur du sang) et ils viennent de "ce continent de la mort qu'on appelle l'Europe" (p. 74). Qu'on ne prenne pas ces images pour de simples illustrations; au contraire, nous avons affaire ici à des essences, qui se manifestent inéluctablement dans le comportement et le caractère des personnages. Les Québécois ne sont jamais plus braconniers que quand ils braquent leurs fusils de chasse sur des hommes, quand ils font ce qui leur est inhabituel en prenant «l'homme pour gibier» (p. 74). Comme Giraudoux, d'après Sartre, Ferron saisit d'abord “l'espèce dans l'individu » et son univers est constitué "d'abord des vérités, d'abord des idées, des significations qui se choisissent elles-mêmes leurs signes ${ }^{14}$ ”. Félix Poutré n'est pas symbolisé par la fourche qu'il tient; cette fourche, impliquant son attachement à la terre et sa petite mentalité de cultivateur, constitue le signe visuel par lequel nous décelons son essence. Sauvageau, cet Amérindien dont le visage nous est encore présenté à la télévision de Radio-Canada pendant les heures où il n'y a pas d'émissions, est l'essence même de la survivance (on n'a pas réussi à l'exterminer, et c'est lui qui remontera les grands soleils «aux quatre coins du pays» (p. 109), et de la sagesse (c'est lui qui révèle à Chénier la nécessité de la mort, de la défaite, pour qu'il y ait une renaissance); de plus, c'est lui qui, fidèle à la légende, apporte le sac contenant les enfants des Canadiens français, "sauvant ainsi son âme en nous la transmettant", dit Mithridate (p. 16). Comme le fait remarquer Michelle Lavoie, “les Canadiens français doivent se reconnaître comme fils de Sauvageau, c'est-à-dire héritiers des Indiens; ... ils doivent reconnaître le visage de Sauvageau sous les traits de Saint Jean-Baptiste ${ }^{15}$ ". Même le nom Sauvageau a ceci de particulier que, tout en contenant le mot "sauvage", il est un nom de famille très répandu au Québec.

Mais c'est de loin les trois personnages principaux de la pièce Mithridate, Élizabeth et Chénier - qui révèlent le plus clairement l'importance des essences dans la composition des personnages. Prenons d'abord Mithridate, ce Roi du Pont qui boit tant de "robine" qu'il ne sait plus de quel pont il s'agit (p. 16). Ce qui distingue ce personnage bien québécois de ses prédécesseurs asiatiques et raciniens n'est pas seulement son inaction et son manque complet de mentalité guerrière, c'est que ce Mithridate est avant tout roi de la parole. Comme son homonyme du conte "Cadieu", Mithridate est "un robineux qui n'aimait guère la robine, intoxiqué surtout de mots ${ }^{16}$. C'est lui le metteur en scène de la pièce, le conteur de la bataille de Saint-Eustache. Il est à la fois la conscience des Québécois (dans sa critique de la participation de François Poutré dans des guerres colonialistes $d u X X^{e}$ siècle) et celui qui leur annonce, à la fin de la pièce, leur victoire future: "le pays est à nous" 
(p. 105). Le génie de Mithridate est sa passion de parler, de dire tout haut ce que les autres n'osent pas dire. Ainsi, au quatrième acte, il verra à travers les apparences et exprimera sa déception face aux événements qui ont suivi la mort de Chénier. Pendant la bataille de Saint-Eustache,

on était tous... des partisans [dit-il]. On a formé un peuple de partisans masqués. Et puis, après tant d'effort et de complicité, au bout de l'obstination de ce peuple à se donner un pays pour arracher ses masques, quand de ta gloire retrouvée naît enfin ta victoire, qu'estce qu'on voit: de tous bords, tous côtés, tes partisans s'égailler pour devenir des, propriétaires et des particuliers! Des particuliers, tu t'imagines ! Étais-tu un particulier, toi, Chénier? (p. 106)

Mais, en fait, Chénier était bei et bien un particulier avant de devenir statue, et la pureté de Mithridate ne peut résister à la gaité de tous ces «particuliers" qui veulent célébrer trop hâtivement une victoire qui n'en est pas une. Mithridate aura droit toutefois aux derniers mots de la pièce, et ces mots résumeront ce trait de caractère qui le définit tout entier: "Ah! lls n'ont pas fini de voir l'oiseau moqueur se tirer de la gueule du chien à trois yeux.» (p. 109) Mithridate, c'est le jusqu'auboutiste; pour reprendre les mots de Ferron lui-même, c'est un personnage «inquiétant " qui "représente la liberté17". On pourrait dire aussi que Mithridate est motivé uniquement par la passion de la liberté pure, si pure qu'elle n'existera jamais qu'en théorie; voilà la raison de son ivresse, 'de sa préférence pour la couleur noire. Il est parfaitement incapable de s'adapter à la vie réelle, et il aurait préféré empailler ses amis (par exemple, François Poutré) au moment de la bataille de Saint-Eustache afin qu'ils restent tranquilles (c'est-à-dire purs) à tout jamais (p. 97). Mais la pureté même de ses passions fait qu'il est le seul personnage, avec Sauvageau, à pénétrer les masques des autres personnages et à se prendre exactement pour ce qu'il est: "Mithridate est mon nom et je me prends pour Mithridate. Je suis roi. Je règne sur moi-même." (p. 96)

Le caractère d'Élizabeth, cette Anglaise devenue Québécoise, me semble aussi univoque, aussi pur que celui de Mithridate. Elle partage avec le robineux certaines caractéristiques, dont la plus importante est celle de voir dans l'avenir: c'est elle qui prédit la fin tragique de Chénier. Elle semble faire partie d'un groupe de gens dont l'origine est incertaine (Sauvageau et Mithridate étant les deux autres membres de ce groupe): ses parents sont morts en mer et les ursulines l'ont recueillie à l'âge de quatre ans (p. 45). Elle est donc une étrangère, mais en même temps, comme le lui affirme le curé, «je crains... que vous ne soyez plus Canadienne qu'il ne faut" (p. 45). Comme Mithridate et Sauvageau, Élizabeth incarne l'essence de la «québécité " (pour emprunter un mot de Marcel Rioux), mais cette essence ne lui a pas été transmise par l'hérédité. Au contraire, cette essence est le résultat d'un contact avec la terre québécoise, avec les forces mystérieuses qui font que, d'après Sauvageau, la nature - une nature «québécoise» - reconnaît son identité :

Élizabeth: Je ne suis pas anglaise! ( $\dot{A}$ Sauvageau). N'est-ce pas, Sauvageau, que je ne suis pas anglaise? 
Sauvageau: Mais non, ma prunelle! Regardez: les grands Soleils sont tournés vers vous.

Élizabeth: Vous parlez pour me rassurer. Est-ce que cela veut dire quelque chose?

Sauvageau: Le pays vous voit et vous admire. (p. 42).

Malgré la certitude de Sauvageau, pourtant, Élizabeth ne sera jamais tout à fait sûre de son appartenance au peuple québécois; mais, par ce procédé précieux que j'ai évoqué plus haut, c'est au moment où elle doute le plus de son authenticité qu'elle se révélera le plus purement québécoise:

J'étais folle, j'étais rousse, j'étais d'une autre nation, j'étais d'un autre sexe... Qu'est-ce que j'ai fait? Ah! Qu'est-ce que j'ai fait? Mes idées, c'étaient les tiennes Chénier... Tes idées, je les ai reprises comme des armes et retournées contre toi qui ne m'avait [sic] rien fait, oh! presque rien! Un prétexte pour mettre fin à un impossible attachement. (p. 86).

Cet attachement "impossible " à Chénier est le double de l'amour de celuici pour sa patrie, et si Élizabeth a "retourné " les idées du docteur «contre" lui, c'était pour que la mort du héros fasse naître un mythe qui aiderait le pays à se faire. Ferron a manifestement voulu que le côté anglais d'Élizabeth soit complètement subordonné à son attachement presque involontaire à sa patrie. Car, comme le reconnaît Mailhot, «à la fin de la pièce elle ne reconnaît ni Mithridate, ni Sauvageau, elle ignore même le nom de Chénier chez qui elle fut servante. Mais lorsqu'enfin elle se souvient, elle revient auprès du sauvage et du robineux, seuls visages authentiques de la Patrie ${ }^{18}$ ».

A côté de ces deux perfections d'essence que sont Mithridate et Élizabeth, Chénier peut sembler au premier abord un personnage imparfait, humain. Lorsqu'on y regarde de près, pourtant, on voit que ce personnage est aussi parfait à sa manière que les deux autres. C'est que chez Chénier on voit le mécanisme de son identification à une essence parfaite; à cause du dédoublement du personnage (à la fois homme et statue) les limites de son caractère et de sa liberté d'action sont nettement mises en relief.

Le monument de Chénier est l'aboutissement de sa vie, son destin inévitable. Mais ce destin, Chénier-l'homme ne le voit qu'au milieu du deuxième acte; il se rend compte alors des limites qui se sont imposées à son champ d'action:

Je n'avais pas vu ce monument au bout de ma vie. Tout se résout. Je n'ai plus rien à demander... j'aurais pu être médecin, un simple médecin, traverser la vie avec mon petit portuna à la main et disparaître sans bruit comme un honnête homme. (p. 66)

Chénier n'a aucun choix: même s'il avait voulu se contenter d'un "soleil immédiat" ( $p$. 77) il est condamné à voir mourir et fleurir des milliers de grands soleils chaque fois que son nom sera invoqué par les Québécois. Mais Chénier n'est pas tout à fait impuissant devant son avenir, car il faudra, pendant cette scène dix du deuxième acte que je viens de citer, 
qu'il choisisse de suivre son destin. Encore une fois les propos de Sartre portant sur Giraudoux semblent s'appliquer parfaitement à cette pièce: si le personnage fictif chez Giraudoux est un archétype, c'en est un «qui se réalise à travers la vie humaine par les actes humains ${ }^{19}$ ". Sartre reconnaît donc "un certain genre de liberté" chez ces personnages car "l'homme se conforme volontairement à son archétype, il se choisit perpétuellement tel qu'il est ${ }^{20} \%$. Pour Chénier-l'homme ce choix d'être soimême se fait à partir de sa prise de conscience de son destin (Chénier la statue); "oubliez la médecine ", lui conseille Mithridate, "de quoi auriezvous l'air sur votre piédestal, le portuna à la main? » (p. 66). Quand Chénier aura pris de l'altitude en devenant son monument, il accédera à la clairvoyance d'un Mithridate. II verra dans l'avenir les enfants qui "boivent le lait des fleurs» et il en ressentira "une sorte de bonheur " (p. 67).

Mais dans sa première incarnation (celle d'un homme) Chénier ne plane pas au-dessus des événements; au contraire, son propre est d'être au cœur même de la bataille et de ne pas pouvoir en prédire l'issue. Dans ce sens, il n'est pas sans ressembler à Hector dans La Guerre de Troie n'aura pas lieu. Tout comme le héros giralducien qui est sûr de l'impossibilité d'une guerre, il voit certains signes (le visage de Colborne, son cheval, les lampes qu'on baisse dans les maisons des Québécois) qui semblent indiquer la victoire immédiate de sa cause; tout comme Hector, Chénier se trompe. Mais cette erreur ne fait que confirmer la perfection de son essence de héros québécois, car il est tellement enraciné dans "un pays qui est nôtre, qui nous enveloppe de sa sympathie» (p. 74) qu'il est incapable de douter de la résistance de tous les Québécois aux Anglais, ou de se voir autrement que "sous l'arc-en-ciel de la victoire" (p. 75). Si donc Mithridate est la conscience du peuple québécois, Chénier est leur idéal:

II s'en remis [sic] à son cœur qui le guide comme il bat, aveuglément il goûte la chaleur de son sang

- c'est le cœur et le sang de son pays;

il fait corps avec lui. (p. 76)

Ainsi Chénier, comme les autres personnages de la pièce, atteint à une sorte de perfection dans son essence d'homme imparfait. Tout chez lui est humain; tout chez lui est québécois. Et la québécité de son caractère est d'autant plus claire que Chénier est engagé dans une lutte contre un ennemi dont l'essence est incompatible avec la sienne. Notons bien que cette opposition, cette antithèse entre Québécois et Anglais n'a rien ici d'historique. Elle est fondée plutôt sur une différence de caractère due à la spécificité du contexte nord-américain par opposition à celui de l'Europe. Les Québécois appartiennent à leur pays même si le pays ne leur appartient pas encore; les Anglais, au contraire, viennent d'un vieux continent introduire la guerre et la servitude au Nouveau Monde. Et bien qu'on ne voit aucun Anglais durant la pièce, leur présence se fait sentir par les images que les personnages évoquent à propos d'eux. On a déjà vu plusieurs de ces images; évoquons les plus importantes parmi elles: 
Anglais

«ils ont des uniformes" (p. 74) "la guerre est leur métier" (p. 74) « ce continent de la mort qu'on appelle l'Europe " (p. 74) mourir en terre étrangère une mort difficile

\section{Canadiens}

sans uniforme “braconniers" (p. 74) des "gens de paix» qui «font la guerre pour la première fois (p. 93)

mourir en terre familière: «nos pères y dorment... mourir n'est pas difficile» (p. 74)

On pourrait ajouter un autre élément à cette opposition : le conflit père/ fils, impliquant non seulement le conflit Félix/François Poutré mais aussi celui qui oppose Chénier à ses maîtres anglais et, d'une façon plus générale, celui qui oppose le Christ crucifié à Dieu le Père. Pour Mithridate, "c'est Chénier qui triomphe et avec lui le Fils contre le Père" (p. 101), mais cette victoire est autre que militaire. C'est le triomphe, par la résistance, d'un peuple revendiquant la paix, et dont l'image est la fleur/le soleil/la chaleur, contre les forces qui, partout dans le monde, oppriment les faibles.

On a donc vu que toutes les images et tous les personnages puisent leur substance et leur signification dans l'antithèse centrale de la pièce. Ils affirment leur vie contre la mort, leur pays contre l'envahisseur, leur mode de vie paisible contre la violence. Mais il importe avant tout de signaler le caractère immuable de cette affirmation. Car si la pièce se termine sur une note optimiste, ce n'est pas que l'évolution de l'histoire a apporté la victoire. Au contraire, Ferron, dans le quatrième acte, souligne le caractère inchangeable de la situation (François Poutré est aussi “chouayen" en 1968 que son frère l'était en 1837) et termine la pièce par une "pirouette" digne de Giraudoux. Mithridate a parfaitement raison de se voir «cocu» (p. 106) et de vouloir célébrer "le retour du vaincu, la victoire de l'infâme" (p. 107). La «victoire» en 1968 est tout aussi ambiguë qu'en 1837, et le pays reste toujours un rêve, un idéal à réaliser dans l'avenir. Mithridate, devant une telle évidence, n'a que deux choix: se tuer ou s'accommoder. II choisit la deuxième solution, mais fidèle à son identité il s'intoxique de mots:

Ouida, en place pour la fête! Particuliers, particulières, cabotins, cabotines, propriétaires, robineux... lapins, lapines, patriotes, sauvages, métallurgistes, faune des Amériques rassembles dans l'ordre de la Patrie, $O$ peuple de mon pays, moi, Mithridate, j'ordonne que sans plus tarder on célèbre cette victoire. (p. 108)

Ce que Jean-Marcel Paquette dit à propös des contes de Ferron s'applique parfaitement ici: il nous met en garde contre le «happy ending " et dit que acette conclusion n'a au fond aucune espèce d'importance et ne constitue pas à proprement parler la victoire dont il est question ${ }^{21}$ ".

La préciosité des Grands Soleils nous révèle donc un monde d'oppositions permanentes où nulle solution ne s'impose. Si la pièce n'est pas tout à fait pessimiste, c'est dans la mesure où Chénier, en rencontrant son destin dans l'église en flammes, prouve que la mort d'un homme peut au moins inciter les générations futures à prendre conscience d'elles-mêmes. 


\section{La Tête du roi}

Il y a bien des ressemblances entre les Grands Soleils et la Tête $d u$ roi $^{22}$. On voit la même polyvalence temporelle, mais cette fois la pièce réunit trois périodes dans l'histoire: les révoltes de Riel en 1870 et 1885, la période qui suivit la première guerre mondiale et la conscription, et l'époque contemporaine, en particulier les années soixante, puisque la pièce rappelle le déboulonnage de la statue de Wolfe à Québec en 1963. La pièce compte des personnages qui sont presque le reflet de ceux qu'on a vus dans les Grands Soleils, en particulier une deuxième Élizabeth (fille adoptive même si elle n'est pas d'origine anglaise), un procureur qui s'apparente à Félix Poutré et Taque (ainsi nommé à cause de l'interjection qui le caractérise: «taccaouère!») qui n'est pas sans rappeler Mithridate ${ }^{23}$. On voit également dans cette pièce le même souci des mots, des images répétées (même l'hélianthe réapparaît) qui, toutes, contribuent à la préciosité de l'œuvre.

Mais cette pièce, écrite cinq ans après la première version des Grands Soleils (1958), va encore plus loin dans le développement des essences parfaites. Ici tout s'attache à une situation politique précise, et quand les personnages parlent, ils le font «au pluriel» (p. 142), en impliquant tout un groupe ethnique ou national. De plus, nous voyons en chair et en os un de ces Anglais qui étaient physiquement absents des Grands Soleils, et cette confrontation entre les représentants des deux "peuples fondateurs" permet à Ferron de développer une antithèse particulièrement aiguë.

Pièce moins poétique que les Grands Soleils, la Tête du roi est surtout un grand débat théâtral sur l'attitude à prendre face à une situation insupportable, celle subie depuis presque deux cents ans par les Canadiens français. Les débats politiques éclipsent parfois les images et les symboles, mais ceux-ci existent et ont un caractère aussi précieux que dans les Grands Soleils. C'est sans doute la tête du roi elle-même (décapitation fictive de la statue d'Édouard VII, Square Philips à Montréal) qui domine la pièce. Cette tête, vide, est parfois anodine (c'est " une citrouille [qui] avait poussé sur la pelouse, à l'ombre du monument» [p. 80]); à d'autres moments elle a une valeur nettement politique (elle est placée entre les bustes de Laurier et de Dollard des Ormeaux) et même quasi religieuse (elle se trouve, au quatrième acte, entre deux cierges sur une espèce d'autel). Elle est à la fois le prétexte de toute l'action de la pièce (la pomme que le serpent jette aux pieds du Procureur dans son jardin d'Éden, qui "fout tout par terre» et qui l'oblige à s'interroger «sur mon influence et mon autorité, de même que sur ma ville, mon comté, ma famille, mon pays» [p. 129]), et le symbole du regard des Anglais, ce regard auquel les personnages ne peuvent échapper et qui détermine leur façon de réagir. Mais la préciosité de cet objet se révèle surtout par le contraste qu'il fait avec le chapeau de Louis Riel, porté par Taque, ce «vieux voyageur" (p. 93) qui se serait battu aux côtés du chef manitobain. 
Comme la statue de Chénier, la tête et le chapeau ont une valeur totémique et constituent les restes tangibles de deux idéaux incompatibles. Le chapeau de Riel est attaché au pays (c'est un chapeau de castor) et s'associe à la trahison des autorités anglo-canadiennes qui, en 1870, ne donnaient pas à Riel le temps de mettre «son chapeau de ministre» (p. 87). Symbole avant tout de l'exilé, du faible écrasé par le fort, il confère à celui qui le porte une pureté d'intentions, un jusqu'auboutisme dans le sens de la liberté qui rappelle les attitudes de Mithridate; ainsi, à la fin de la pièce, Taque met le chapeau de Riel et «s'en va ainsi coiffé, droit devant lui, sans un salut, sans un regard pour personne" (p. 138). La tête du roi, zau contraire, est l'incarnation de la force brute, de la domination impitoyable. Entourée de son auréole royale, elle permet à la reine Victoria d'avoir "bonne conscience» en faisant pendre Riel (p. 102). Elle incarne une force historique (une grande machine qui fonctionnait tout seule" [p. 105]) qui existe au-delà de la volonté des hommes («les Windsor eussent été des singes que cela n'y eût rien changé" [p. 105]). L'importance de la tête et du chapeau prend toute son ampleur dans une scène de guignol fascinante: il s'agit de la sixième scène du deuxième acte où la tête et le chapeau s'affrontent physiquement, ayant pour voix le Procureur et son fils Simon.

Les couleurs dans les Grands Soleils variaient du rouge au blanc au vert; dans la Tête du roi elles sont réduites à un contraste de noir et blanc, reflétant ainsi la simplicité de la situation politique de la pièce. Pour la reine Victoria, «si pure, si blanche," les révoltés canadiens sont "noirs et, disons-le, un peu répugnants" (p. 103). Pour Riel, au contraire, le noir est la couleur de la puissance anglaise, dont l'effet tangible est le train transcontinental, tout noir de charbon, «a mari usque ad mare, écrasant tout sur son passage" (p. 103). Entre le noir et le blanc est le gris, couleur par excellence du procureur qui se grise afin de ne pas avoir à prendre parti dans une situation qui, pour lui, est grise - qui n'a pas de contours définis. Et ces couleurs s'attachent à tout un mouvement descendant qui parcourt la pièce et qui fait contraste avec le mouvement ascendant, les "poussées verticales, " les "hommes comme des totems qui montent dans le ciel " qui caractérisent les Grands Soleils (les Grands Soleils, p. 67). Ce n'est pas seulement la tête de la statue qui tombe dans la Tête du roi, c'est tout un peuple, de plus en plus gris parce qu'enivré de l'odeur des fleurs qui poussent dans leurs jardins trop paisibles. Dans un pays gris, "dont la force est l'inertie", nous dit le procureur (p. 128), l'existence est une descente perpétuelle vers la mort: "allons-nous continuer à descendre?... Tout le monde dans l'ascenseur: en bas, going down! Avons-nous encore vie? Nous sommes peut-être morts" (p. 129). Et le seul moyen d'échapper à cette grisaille serait de la couvrir de rouge, de sang; la décollation des statues doit céder, selon le procureur, à la décapitation des hommes:

La violence dans les musées, la décollation d'un roi de bronze, laissezmoi rire un peu! Vous ne dérangez personne, le pays restera inerte. Le vif, au moins si vous cherchiez le vif! ... II faudrait frapper pour 
qu'on saigne au lieu de parler. Le sang, vois-tu, il n'y a rien d'autre qui régenère un peuple! (p. 129)

Mais la Tête du roi ne contient pas la richesse d'images des Grands Soleils. C'est de loin les personnages qui importent ici, et l'antithèse centrale de la pièce est axée, encore plus que dans les Grands Soleils, sur des différences de personnalité, de mode d'être.

D'un côté de cette antithèse se trouvent les Anglais et leurs descendants canadiens. II ne s'agit plus de généraux comme Colborne, et la reine Victoria et son fils ne sont que des marionnettes. L'Anglais qui domine la pièce et qui sera défini comme l'Anglais type, est un Canadien libéral, un partisan de la négociation, de la compréhension, et qui parle «trop bien français" (p. 148). Scott Ewen ${ }^{24}$ est celui dont la mission est de combler les "deux solitudes" de MacLennan, c'est un ami de Pierre (fils du procureur), et pourtant c'est un Anglais qui ne peut, quoi qu'il fasse, échapper à son essence d'Anglais. Dans une scène (acte IV, scène 3) qui ressemble au débat entre Ulysse et Hector à la fin de La Guerre de Troie n'aura pas lieu, Scott Ewen évoque toute la litanie des progressistes canadiens-anglais: le progrès des Québécois, la tolérance, l'accommodation. Mais il a beau essayer de se distinguer de ses ancêtres colonialistes, Simon ne lui pardonnera jamais d'avoir fait remplacer Londres par Ottawa: "vous n'avez plus pour nous que de la condescendance", lui dit-il, "nous n'avons plus pour vous que du ressentiment. Votre domination nous est plus odieuse que ne le fut jamais celle de l'Angleterre» (p. 142). Devant une telle affirmation, Scott ne peut que se rendre à l'évidence. II essaie de fuir le dénouement inévitable de la conversation en invoquant sa liberté d'individu: "Je suis seul ici, je ne parle qu'en mon nom," dit-il (p. 142) et plus tard: “Voudriez-vous que je me sente coupable de la mort de Jeanne d'Arc?" (p. 142) Mais ces propos mêmes prouvent qu'il parle au nom de tous les Anglais:

Scott : Que voulez-vous dire au juste?

Simon: Que le Québec soit mon pays et que dans mon pays je sois chez moi.

Scott: Eh bien! Soyons-le et n'en parlons plus.

Simon: A la condition, bien sûr, de ne pas vous déranger. Et s'il fallait que nous vous dérangions un peu?

Scott: Je vous répondrai franchement: dérangez-nous le moins possible. (p. 142)

On pourrait dire de Scott Ewen qu'il n'est jamais plus anglais que quand il parle français.

Ferron va très loin ici dans sa définition du caractère anglais. Tout ce que font les Anglais les stigmatise, et l'on se rappelle encore une fois le Siegfried de Giraudoux où les plus petits détails de la vie avaient un caractère allemand ou français. En effet, ce n'est pas la langue qui, chez Ferron, distingue le Canadien anglais du Québécois; Scott Ewen a raison de dire: "la différence entre nos deux langues est si petite! C'est même une pitié que d'avoir à nous affronter pour elle» (p. 143). Mais Simon ré- 
plique: "La langue n'est qu'un prétexte; le véritable enjeu de la lutte est l'honneur de l'homme, qui ne peut se concevoir dans l'abaissement d'un peuple au profit d'un autre." (p. 143) Tout chez les Anglais semble exprimer leur goût de la domination. C'est la veuve du juge Fiset qui met les points sur les $\mathrm{i}$ : si, chez eux, les Anglais sont «des végétariens, des abstinents ", quand ils sont à l'étranger ce sont « de véritables carnassiers" (p. 117). Et ce désir de s'échapper de leur propre puritanisme explique leurs conquêtes:

La Veuve: Ils en avaient soupé d'être puritains et pleins le nez de leurs biscuits; ils en avaient marre de s'ennuyer: ils voulaient vivre. Ils ont bousculé les peuples et les nations. Mais ce sont des gens polis: comme personne ne les avait invités, ils revenaient au-devant d'eux-mêmes pour se souhaiter la bienvenue: "Come in boys! Bifteck, bifteck! " Ils faisaient un bon repas ou deux, puis tout était à recommencer; ils repartaient à l'assaut d'autres pays; ils se sont répandus ainsi par toute la terre. (p. 117)

Si l'explication de la veuve a peu de poids d'un point de vue historique, elle est significative pour la compréhension de l'archétype anglais. Pierre, frère cadet de Simon, qui ne partage pas l'anglophobie de la veuve, explique cet archétype d'une façon moins violente. II est, dit-il, amateur de cimetières anglais puisque "ce sont les plus beaux parcs... II y a dedans profusion de fleurs, de buissons, de bosquets, et, çà et là, solitaires, des saules pleureurs" (p. 119). C'est le saule pleureur qui, pour Pierre, incarne le caractère anglais: ces arbres

ne pleurent pas vraiment. La lumière coule sur leurs épaules tombantes. Une grande mante radieuse double leur mante verte. Leur tête a fondu dans le ciel, il n'en reste que le sourire, mais c'est bien le plus beau sourire du monde. (p. 119)

À ce sourire éternel, à cette timidité apparente qui cache si bien l'agressivité, le pauvre Scott Ewen, qui «s'intéresse aux insectes, aux petits mammifères, aux oiseaux", ( $p$. 121) ne peut échapper. Taque croit voir dans son visage un policier (celui qui a arrêté Riel), et cela suffit pour pousser ce Canadien errant à prendre la fuite. Élizabeth est assez clairvoyante pour comprendre le départ de Taque; elle explique à Scott: «il avait vu vos devanciers, Monsieur, à l'œuvre au milieu de peuples en perdition, de peuples aujourd'hui disparus; et il a trouvé que vous leur ressembliez, vous n'y pouvez rien» (p. 147). C'est en fin de compte le visage de Scott qui, plus encore que ses propos, le trahit et l'expose. Nous sommes encore une fois dans cette ambiance de bal masqué dont parlait Claude-Edmonde Magny, où l'apparence est la clé à la compréhension de l'être.

On peut qualifier tous les personnages de la Tête du roi d'archétypes. Ainsi, par exemple, Émond (Louis Hémon?), valet français du procureur, est à mi-chemin entre les Anglais et les Québécois. Apologiste du colonialisme, il soutient néanmoins la libération des peuples asservis car "l'organisation du monde ne peut qu'aboutir à l'égalité de tous les hommes" (p. 104). Il salue donc "et le pendu et le décapité", Riel et le 
roi anglais (p. 110). Comme De Gaulle, il s'intéresse à la sécession du Québec, «non pour libérer ce pauvre petit pays, mais pour mieux l'incorporer à son empire" (p. 110). Ses «néveurmagne" témoignent de son désir de parler comme les Québécois mais il n'a pas encore l'accent et, d'après Taque, il lui faudra «deux ou trois générations " avant de l'acquérir (p. 68). Bref, Émond, comme Scott, ne peut échapper à son essence; c'est "le Français qui a fui la France et que la France n'a point quitté" (p. 122).

De même, les femmes ici sont la quintessence de la féminité, parfois agressive (la veuve), parfois coquette (Élizabeth), mais toujours motivée par l'amour et toujours soumise à l'autorité masculine. Comme dans les pièces de Giraudoux, les femmes ont un statut spécial; ce sont elles qui, comme l'Élizabeth des Grands Soleils, encouragent les hommes et semblent avoir un sixième sens qui leur permet de voir clair là où les hommes ne voient que du feu. N'oublions pas que c'est Élizabeth qui, par sa clairvoyance de jeune fille, provoque le dénouement de l'intrigue. En courant après Taque qui fuyait, en se sentant humiliée parce qu'elle croyait que son père l'était, mais surtout en réagissant d'une façon si honnête à Scott Ewen et en lui demandant s'il venait «à titre d'ami ou d'envahisseur" (p. 147), question à laquelle l'Anglais refuse de répondre, Élizabeth mérite le respect et l'admiration du procureur:

S'il y a quelque chose de changé dans cette maison, dans ce pays, c'est à toi que nous le devons. II fallait une réaction simple et spontanée, une réaction de jeune fille, pour en finir une fois pour toutes avec le malaise où nous étions. (p. 151)

Quant à Taque, au procureur et à ses deux fils, ils incarnent l'essence québécoise dans toute sa complexité. C'est surtout la situation du procureur qui est intéressante: il semble résumer à lui seul le dilemme moral qui est à la base de la pièce. Dans ce contexte, ses deux fils ne sont que le reflet du tiraillement qui s'exerce sur lui: Simon, comme son homonyme biblique si avide de miracles, veut conférer à la tête du roi le pouvoir magique de tout changer au Québec, tandis que Pierre, poète planant au-dessus de la situation, acteur par excellence, joue un rôle selon la direction du vent, puisqu'il est «lié aux deux partis" (p. 122). Comme il a pu élever deux fils si différents, le procureur a pu s'accommoder à ses contradictions, «être rebelle et loyaliste, cumuler les fonctions de serviteur de la Couronne et de notable nationaliste" (p. 149). Le problème du procureur, c'est très littéralement une incapacité de se prononcer: il avait perdu la main de la veuve (avant son mariage au juge Fiset) parce qu'il n'avait pas osé lui expliquer la différence entre un étalon et une jument (p. 92). Appelé à remplacer le défunt juge, il se voit soudain compromis dans sa carrière par la présence de la tête du-roi dans sa maison. Que fera le procureur? Dénoncera-t-il ceux qui ont décapité la statue, en l'occurrence son propre fils Simon? Passera-t-il du côté des révolutionnaires? 
Comme les Grands Soleils, la Tête du roi se termine par une pirouette. Jusqu'à l'arrivée de Scott Ewen, le procureur semble aller de plus en plus vers une sympathie ouverte envers les révolutionnaires nationalistes. II accepte volontiers qu'on le traite de coupable, revendiquant pour lui la guillotine de Riel (p. 110) et la couleur noire, symbole, dit-il, de sa culpabilité envers le pouvoir établi (p. 114). Il crie aux Anglais (absents): "Boss, on ne te dit rien, tu nous achales et je t'emmerde! Boss, garde tes distances, éloigne-toi, encore un peu, et puis continue donc et va bosser ailleurs... Dorénavant tu ne nous constipes plus." (p. 114) Quand Simon doute de la sincérité de ces propos, accusant son père de faire du théâtre, le procureur réplique: «j'ai pris conscience de moi-même » (p. 128). Mais la présence de Scott Ewen change tout. Ses yeux anglais, fixés sur le procureur, le mettent manifestement aussi mal à l'aise que l'était Taque, et surtout ils lui rappellent sa situation de colonisé, d'inférieur. Et nous voici donc devant le point d'interrogation de la pièce. Le procureur s'est-il grisé afin de ne pas dire «ce qu'il fallait dire et que nous attendions tous" devant Scott Ewen? (p. 136). A-t-il ainsi humilié son peuple 25 ? Ou le procureur dit-il vrai en affirmant avoir feint l'ivresse afin de ne pas "gâter cette longue conquête par un geste inconsidéré »? (p. 151). Peut-on le croire quand il affirme: «je n'hésiterai plus entre le noir et le blanc»? (p. 151) Ferron nous laisse délibérément devant cette ambiguiité. Le procureur, en tout cas, a été fidèle à son essence de procureur: l'ivresse, vraie ou feinte, lui a permis de remettre encore davantage la décision sur laquelle il ne pourra pas revenir. Remarquons du moins une petite victoire pour les nationalistes: la tête du roi trouve sa place définitive à la fin de la pièce non pas comme symbole de l'autorité anglaise mais comme un simple vase rempli de fleurs. Mais même cette victoire est diluée par la certitude qu'une des nombreuses «pièces de rechange" que les autorités gardent pour de telles éventualités a permis à la statue du roi d'avoir une nouvelle tête (p. 145). Si la pièce se termine donc par l'évocation d'un pays enfin libre, où l'Anglais n'entrera qu'à titre d'invité, cet avenir n'est que très hypothétique.

\section{CONCLUSION}

Pourquoi un écrivain du $x x^{\ominus}$ siècle s'exprimerait-il d'une façon précieuse? Quel est le but de la préciosité dans la littérature moderne? Pour Claude-Edmonde Magny, la littérature précieuse aurait «une fonction compensatrice ${ }^{26}$ " en ce qu'elle fournirait au public «un certain confort intérieur $^{27}$ ". A certaines époques dans l'histoire (dont la nôtre) où les valeurs traditionellement acceptées sont en train d'éclater, le public a besoin, nous dit-elle, d'un "monde d'archétypes... où les choses s'accordent à l'idée que nous avons d'elles sans jamais nous inquiéter ou nous surprendre ${ }^{28}$.

Mais ce qui vaut pour Giraudoux ne vaut pas forcément pour Ferron. Si Ferron partage certaines techniques avec Giraudoux, sa vision du 
monde politique diffère sensiblement de celle du diplomate français. Pourquoi Ferron a-t-il choisi le mode précieux pour ses pièces politiques? Quel est l'apport de la préciosité à la formation des idées exprimées dans ses pièces? Sans prétendre donner à ces questions une réponse définitive, j'essaierai d'apporter quelques précisions.

Le caractère antithétique des rapports 'entre les groupes sociaux a sans doute un rôle très important à jouer dans la vision politique de Ferron. Dans un monde composé d'archétypes qui s'affrontent et se confrontent, comment arriver à un compromis entre des gens qui diffèrent essentiellement? II semble en particulier que pour Ferron, le caractère authentiquement nord-américain (comprenant les Amérindiens aussi bien que les Québécois) et le caractère européen aient très peu en commun. Taque, par exemple, se défend d'être qualifié d'anarchiste par Émond, trouvant que cette appellation européenne convient peu au contexte canadien (la Tête du roi, p. 69). D'ailleurs, dans son "Adieu au P.S.D." écrit en 1960, Ferron avait mis les Québécois en garde contre «l'influence européenne" et "les Canadiens sans racine qui imposent leurs ramures aux vieilles souches 29 ". Les Français, en particulier, auraient un caractère essentiellement incompatible avec les intérêts des Québécois. Émond, par exemple, voudrait que les Québécois, au lieu de saluer la reine, crient "vive la France," mais le procureur se rend compte du danger de cette situation qui aurait l'effet de remplacer la potence par la guillotine, "automatique et tricolore" (la Tête du roi, p. 111). Du point de vue politique, on peut dire avec Taque que "les Français des vieux pays sont des sortes d'Anglais» ( $p$. 107) qui ne pourront jamais avoir les mêmes idéaux que ce "premier peuple blanc qui cède au métissage et se lève avec le TiersMonde" (les Grands Soleils, p. 101). Quant aux Anglais, contentons-nous de citer ce passage de l' “Adieu au P.S.D." qui pourrait se rapporter précisément à Scott Ewen :

Le socialisme de nos compatriotes anglais n'est qu'un masque pour continuer la seule politique qu'ils aient jamais eue au Canada: imposer leur domination, catchup on steak coast to coast. Là-dessus, ils ne transigent jamais ${ }^{30}$.

Devant de tels propos, certains vont crier au racisme. Mais ce serait mal comprendre Ferron que d'assimiler ses idées aux nationalismes de droite qui ne confèrent qu'aux catholiques d'origine française le droit de s'appeler Québécois. Au contraire, comme la situation d'Ellizabeth dans les Grands Soleils le montre, le Québécois est celui qui, de quelque origine qu'il soit, fait cause commune avec la majorité française du pays. Mais faire cause commune n'est pas chose simple; ce n'est pas en se prononçant en faveur du bilinguisme (comme le fait Scott Ewen) qu'on devient québécois. La québécité s'acquiert par le contact avec le pays - avec la terre, certes, mais surtout avec le peuple - et par la volonté de donner sa vie pour une cause. Ferron suppose que quand la guerre éclatera (si jamais elle éclate) ceux qui seront aux côtés des Québécois auront fait preuve de leur essence québécoise. En attendant, ce sont les Haïtiens qui 
meurent de faim, les Vietnamiens dans leur pays dévasté, les Africains écrasés par le racisme qui sont les alliés naturels du peuple québécois.

Mais encore plus que l'affrontement d'archétypes, le caractère essentiellement théatral de la préciosité de Ferron confère un sens politique à ses deux pièces. On sait que la Tête du roi a lieu pendant la Fête-Dieu et que les fêtes de la victoire jouent un rôle important dans les Grands Soleils. Mais ce qui frappe dans le théâtre politique de Ferron, c'est que toute action est cérémoniale, théâtrale. Les Grands Soleils sont une espèce de rite où Mithridate, après avoir dirigé une "scène préliminaire d'exorcisme», lit les "Nolets" qui racontent l'histoire tandis que les personnages répètent, au présent, des mots qu'ils ont prononcés il y a plus de cent ans. La Tête du roi contient plusieurs scènes de théâtre interne, dont celle des marionnettes (dont j'ai parlé plus haut); d'ailleurs, d'après Émond, toute la pièce est une série de guignols (/a Tête du roi, p. 129).

Nous pouvons voir dans la Tête du roi la vraie signification de ces jeux. Car le goût du procureur pour de petites scènes de théâtre s'explique par son désespoir. Homme sans pays, serviteur chez lui, son existence est précaire; afin de pouvoir vivre il est obligé de se donner plusieurs identités; il est tantôt loyaliste, tantôt indépendantiste. Dans un pays où «il n'y a plus rien de sacré, rien sauf les gros sous" et où «la reine est dans le lit du banquier» (p. 126), comment donner aux mots et aux gestes une signification? Voilà pourquoi le procureur ne prend pas le stratagème de Simon au sérieux: la tête du roi n'a que très peu d'importance dans le Canada d'aujourd'hui car le vrai pouvoir n'est plus chez la royauté. "Un seul assassinat pour prouver que vous êtes sérieux!" demande le procureur à son fils (p. 129). Mais même cette colère n'est que du simulacre, et Émond clôt la discussion en bon metteur en scène: "ce sera pour le prochain guignol, après celui de l'Anglais" (p. 129).

Dans un pays qui n'est qu'un rêve, «comment réussir une révolution si l'on n'est pas poète? " demande le procureur; “il faut prendre de l'altitude», est sa réponse (p. 128). Jusqu'au moment où le pays existera, tout ce qui se passe au Québec échappera aux Québécois, tout sera jeu, simulacre. On jouera le jeu, quitte à se griser (ou à faire semblant) afin de "prendre de l'altitude " face à la réalité.

On voit donc que la préciosité chez Ferron, au contraire du théâtre de Giraudoux, sert à faire réfléchir plutôt qu'à rassurer. Ferron nous place devant une situation fixe, immuable; il nous demande de prendre parti, de prendre conscience de nous-mêmes. Mais au moment final, où nous lui demandons de nous aider à trouver la certitude que nous recherchons, il s'éclipse. Et il nous laisse seuls à méditer ces mots de Simon dans la Tête du roi (p. 143): "il s'agit de deux choses l'une, du dernier sursaut d'un peuple vaincu ou du début de sa libération ". 
1. On sait que cette division chronologique a été fortement contestée par JeanMarcel Paquette dans un article intitulé "Jacques Ferron ou le drame de la théâtralité " auquel j'aurai l'occasion de me référer plus loin.

2. Voir André Vanasse, "le Théâtre de Jacques Ferron à la recherche d'une identité, » dans Livres et auteurs québécois, 1969, p. 226 et 229.

3. Guy Beaulne, «la Tête du roi de Jacques Ferron, " dans Livres et auteurs canadiens, 1963, p. 42.

4. Jean-Marcel Paquette, "Jacques Ferron ou le drame de la théâtralité," dans le Théatre canadien-francais, tome $\mathrm{V}$ des Archives des lettres canadiennes, Montréal, Fides, 1976, p. 592 et 593.

5. Michelle Lavoie, «Jacques Ferron ou le prestige du verbe, dans Études françaises, vol. $V, n^{\circ} 2,1969$, p. 187.

6. Paquette (article cité, p. 593), emploie cette expression et Michelle Lavoie (article cité, p. 187) parle de la façon "parabolique" dont s'expriment les personnages des pièces de Ferron.

7. Claude-Edmonde Magny, Précieux Giraudoux, Paris, Le Seuil, 1945, p. 31. Toutes les citations qui suivent renvoient à cette édition.

8. Jean-Paul Sartre. "M. Jean Giraudoux et la philosophie d'Aristote ", dans Situations I, Paris, Gallimard, 1947, p. 76-91. Toutes les citations qui suivent renvoient à cette édition.

9. Jacques Ferron, les Grands Soleils, dans Théâtre I, Montréal, Déom, 1969. Toutes les citations qui suivent renvoient à cette édition.

10. Laurent Mailhot, "Jacques Ferron: de l'amour incertain à la patrie possible", dans J.-Cléo Godin et Laurent Mailhot, le Théâtre québécois, Montréal, HMH, 1970 , p. 167.

11. Claude-Edmonde Magny, ouvrage citè, p. 117.

12. Jean-Marcel Paquette, article cité, p. 590.

13. Voir également Donald Smith, "Jacques Ferron ou la folie d'écrire», dans les Lettres québécoises, $n^{\circ} 6$, avril-mai 1977, p. 40.

14. Sartre, article cité, p. 78 .

15. Michelle Lavoie, "Jacques Ferron de l'amour du pays à la définition de la patrie," dans les Cahiers de Sainte-Marie, $n^{\circ} 4$, p. 99-100, citée par Laurent Mailhot, p. 164

16. Jacques Ferron, Contes, Montréal, HMH, 1968, p. 21.

17. Jacques Ferron dans le Devoir, 14 mai 1968, article reproduit dans Théatre I, p. 121.

18. Laurent Mailhot, article cité, p. 165.

19. Sartre, article cité, p. 87.

20. Ibid., p. 87

21. Jean Marcel [Paquette], Jacques Ferron malgré lui, Montréal, Éditions du Jour, 1970, p. 192.

22. Jacques Ferron, la Tête du roi, dans Theâtre II, Montréal, Déom, 1975. Toutes les citations qui suivent renvoient à cette édition.

23. Voir Laurent Mailhot, article cité, p. 167.

24. On sait que Ferron a dédié cette pièce au journaliste et romancier torontois Scott Symon, et il est permis de se demander si le débat du quatrième acte qui oppose Scott et Simon est une coïncidence.

25. Je note ici, à titre personnel, une attitude que je partageais avec d'autres Juifs pendant mon enfance: toute humiliation d'un membre du groupe ethnique devant un "Gentil" était ressentie comme l'humiliation de la communauté juive tout entière.

26. Claude-Edmonde Magny, ouvrage cité, p. 48.

27. Ibid., p. 49.

28. Ibid., p. 50.

29. Jacques Ferron, "Adieu au P.S.D.", dans la Revue socialiste, été 1960, reproduit par Jacques de Roussan, Jacques Ferron, quatre itinéraires, Montréal, Les Presses de l'Université du Québec, 1971, p. 35.

30. Ibid., p. 34 